\title{
Microesferas magnéticas à base de poli (metacrilato de metila-co-divinilbenzeno) obtidas por polimerização em suspensão
}

\section{Magnetic microspheres based on poly(divinylbenzene-co-methyl methacrylate) obtained by suspension polymerization}

\author{
Jacira Aparecida Castanharo ${ }^{1}$, Ivana Lourenço de Mello Ferreira ${ }^{1}$, Marcos Antonio da Silva Costa ${ }^{1}$, \\ Manoel Ribeiro da Silva², Geraldo Magela da Costa $^{3}$ e Márcia Gomes de Oliveira ${ }^{4}$
}

\author{
1 Laboratório de Química de Polímeros, Instituto de Química, \\ Universidade do Estado do Rio de Janeiro - UERJ, Rio de Janeiro, RJ, Brasil \\ ${ }^{2}$ Departamento de Física e Química, Instituto de Ciências, Universidade Federal de Itajubá - UNIFEI, \\ Itajubá, MG, Brasil \\ ${ }^{3}$ Departamento de Química, Universidade Federal de Ouro Preto - UFOP, Ouro Preto, MG, Brasil \\ ${ }^{4}$ Divisão de Processamento e Caracterização de Materiais, Instituto Nacional de Tecnologia - INT, \\ Rio de Janeiro, RJ, Brasil \\ *marcoscosta.iq.uerj@gmail.com
}

\begin{abstract}
Resumo
Microesferas poliméricas à base de metacrilato de metila (MMA), divinilbenzeno (DVB) e material magnético foram preparadas via polimerização por suspensão. Foi estudada a influência da polimerização em suspensão ou semisuspensão, concentração de material magnético e concentração de divinilbenzeno sobre as características das microesferas obtidas. As partículas poliméricas foram caracterizadas por espectroscopia vibracional na região do infravermelho por transformada de Fourier (FT-IR), microscopia eletrônica de varredura, analisador de área específica e porosimetria, espalhamento de luz e magnetometria de amostra vibrante. O material magnético também foi caracterizado por FT-IR, além de difratometria de raios X e espectroscopia Mössbauer. Foram obtidas, com sucesso, microesferas poliméricas magnéticas à base de MMA e DVB (P(MMA-co-DVB)-M), contendo partículas magnéticas tanto na superfície quanto no interior da microesfera. As partículas obtidas através de polimerização em semisuspensão apresentaram tamanhos menores e distribuição de tamanhos de partículas mais estreita que as partículas obtidas através de suspensões convencionais. Os compósitos apresentaram um comportamento superparamagnético. As microesferas magnéticas sintetizadas neste trabalho têm potencial para serem modificadas e aplicadas como resinas de troca iônica ou suportes catalíticos.
\end{abstract}

Palavras-chave: polimerização em suspensão, propriedades magnéticas, metacrilato de metila, divinilbenzeno, material magnético.

\section{Abstract}

Magnetic polymeric microspheres based on methyl methacrylate (MMA), divinylbenzene (DVB) and magnetic materials were prepared via suspension polymerization. We studied the influence of the suspension or semi-suspension polymerization, magnetic materials and divinylbenzene concentration on the characteristics of the microspheres. Polymeric particles were characterized by Fourier transform infrared spectroscopy (FT-IR), scanning electron microscopy, accelerated surface area and porosimetry analyzer (ASAP), dynamic light scattering (DLS) and vibrating sample magnetometer (VSM). The magnetic materials were also characterized by FT-IR, X-ray diffraction and Mössbauer spectroscopy. Magnetic polymeric microspheres based on MMA and DVB (P(MMA-co-DVB)-M), containing magnetic particles on the surface or inside the microspheres, were successfully obtained. The polymer particles obtained through semi-suspension polymerization had smaller sizes and narrower particle size distribution than particles obtained by conventional suspension. The composites showed a superparamagnetic behavior. The magnetic microspheres synthesized in this work have potential to be modified and applied as ion-exchange resins or catalyst supports.

Keywords: suspension polymerization, magnetic properties, methyl methacrylate, divinylbenzene, magnetic materials. 


\section{Introdução}

A aplicação de microesferas poliméricas magnéticas em processos de separação deve-se a sua fração inorgânica, composta essencialmente do material magnético que é responsável pela resposta ao campo magnético aplicado. A introdução do material magnético facilita a separação das microesferas poliméricas do meio de aplicação de um campo magnético externo. Recentemente, tem sido descrita na literatura a preparação de microesferas poliméricas magnéticas à base de metacritalato de metila (MMA). Ma et al.$^{[1]}$ sintetizaram, com alta agitação, microesferas não porosas, à base MMA, peróxido de benzoíla (BPO) como iniciador e magnetita $\left(\mathrm{Fe}_{3} \mathrm{O}_{4}\right)$. Posteriormente, as microesferas foram modificadas com etilenodiamina para introdução de íns $\mathrm{Cu}^{2+}$. $\mathrm{O}$ carregamento com estes cátions resultou em microesferas poliméricas magnéticas capazes de ancorar proteínas que possuíam afinidade com o metal. Yang et al. ${ }^{[2]}$ também prepararam copolímeros à base de MMA, utilizando $\mathrm{BPO}$ como iniciador, na presença de $\mathrm{Fe}_{3} \mathrm{O}_{4}$ pela técnica de suspensão via pulverização. Um ensaio de adsorção com albumina indicou que estas microesferas magnéticas podiam melhorar significativamente a capacidade de adsorção de proteínas. Apesar de estes trabalhos apresentarem materiais com morfologia esférica, boas magnetizações de saturação (15-17 emu/g) e tamanhos de partículas menores $(1-10 \mu \mathrm{m})$ que as obtidas em processos de suspensão convencionais, esses estudos se concentraram mais na aplicação destas microesferas poliméricas magnéticas. Não foi realizado um estudo sobre a influência dos parâmetros de polimerização sobre as propriedades das partículas poliméricas magnéticas obtidas.

O uso da técnica de semissuspensão na síntese de microesferas poliméricas magnéticas também tem sido descrito na literatura ${ }^{[3-5]}$. Esta técnica permite que curvas de distribuição de tamanho de partículas mais estreitas sejam obtidas. A técnica consiste numa polimerização em suspensão em duas etapas. Inicialmente é feita uma polimerização em massa e quando a conversão alcança um determinado valor (por exemplo, $25-30 \%$ ), a mistura reacional altamente viscosa é transferida para um reator que contém água e estabilizante, onde a polimerização prossegue até que se alcance a conversão desejada ${ }^{[4]}$.

Em nosso grupo de estudo, Costa et al. ${ }^{[3]} \mathrm{e}$ Souza et al. ${ }^{[6]}$ também sintetizaram copolímeros magnéticos à base de MMA via polimerização em semissuspensão e suspensão. Em ambos os trabalhos foram utilizados BPO, gelatina como agente de suspensão e como material magnético ferro recoberto com 5-10\% de óxido de ferro (Carbonyl Iron Powder OX - Basf). Embora os autores tenham sintetizado as microesferas magnéticas com sucesso, o material magnético utilizado nestes trabalhos possui tamanho de partículas maior $(4 \mu \mathrm{m})$ do que a magnetita e a maghemita $(8-30 \mathrm{~nm}$, por co-precipitação) e, por isso, apresentam uma menor susceptibilidade magnética ${ }^{[7,8]}$. Além disso, a distribuição de tamanho de partículas das microesferas poliméricas foi larga $(75-700 \mu \mathrm{m})$. Esta característica limita o emprego destes materiais em processos de separação, pois as reações de superfície são beneficiadas em função da maior área especifica das microesferas que são obtidas quanto menor for o tamanho das partículas.
Há relatos na literatura de que, assim como o uso da semissuspensão, o uso do iniciador AIBN e do agente estabilizante poli(álcool vinílico)(PVA) colaboram para que o tamanho médio de partículas diminua e a curva de distribuição de tamanho médio de partículas fique mais estreita $^{[4,59,10]}$. Além disso, já é bem conhecido na literatura que microesferas poliméricas magnéticas podem apresentar estado de superparamagnetismo com o uso de maghemita $\left(\mathrm{Fe}_{2} \mathrm{O}_{3}\right)$ ou magnetita $\left(\mathrm{Fe}_{3} \mathrm{O}_{4}\right)^{[8,11]}$. Sendo assim, o objetivo deste trabalho foi sintetizar microesferas magnéticas à base de MMA e DVB, utilizando AIBN como iniciador e PVA como agente de suspensão. Foram investigados os efeitos da utilização das técnicas de suspensão e semissuspensão, bem como das concentrações de material magnético e de divinilbenzeno sobre as propriedades das microesferas poliméricas magnéticas obtidas.

\section{Metodologia}

\subsection{Preparação do material magnético}

A síntese do material magnético foi realizada pela técnica de co-precipitação baseada nos trabalhos de Koneracká et al. ${ }^{[1]]}$ e Guo et al. ${ }^{[12]}$ com modificações. Em dois béqueres de $100 \mathrm{~mL}$ foram pesados $27 \mathrm{~g}$ de cloreto férrico $\left(\mathrm{FeCl}_{3}\right)$ (Vetec, RJ, Brasil) e $14 \mathrm{~g}$ de sulfato ferroso $\left(\mathrm{FeSO}_{4}\right)$ (Vetec, RJ, Brasil). Foram adicionados $50 \mathrm{~mL}$ de água deionizada em cada um para a dissolução completa dos sais. Em seguida, as soluções foram misturadas com auxílio de agitação mecânica (300 rpm) em atmosfera de nitrogênio e em temperatura ambiente obtendo-se uma solução de coloração avermelhada. Ainda em temperatura ambiente e atmosfera de nitrogênio, foi adicionada lentamente à mistura $130 \mathrm{~mL}$ de solução de hidróxido de amônio $\left(\mathrm{NH}_{4} \mathrm{OH}\right)$ (Vetec, $\mathrm{RJ}$, Brasil). Após o término da adição a temperatura foi elevada a $70^{\circ} \mathrm{C}$ e mantida nesta temperatura por 1 hora. Após esta etapa, o precipitado preto obtido foi lavado diversas vezes com água deionizada até que o $\mathrm{pH}$ do sobrenadante ficasse em torno de 7. Em seguida adicionou-se 2 gotas de $\mathrm{HCl}$ (Vetec, RJ, Brasil). Posteriormente, $10 \mathrm{~mL}$ de ácido oleico (B'Herzog, RJ, Brasil) foi adicionado sob agitação branda e sem atmosfera de nitrogênio numa temperatura de $80^{\circ} \mathrm{C}$. Após o término da adição do ácido oleico, a mistura reacional permaneceu nesta temperatura por 30 minutos. Então, a mistura final foi lavada com etanol diversas vezes para a retirada do excesso de ácido oleico. Em seguida o material em lama foi transferido para um dessecador, onde o ar foi evacuado e substituído por nitrogênio. Este procedimento foi repetido a cada utilização do material magnético.

\subsection{Preparação das resinas poliméricas magnéticas}

A fase orgânica (FO) foi constituída de metacrilato de metila (MMA) (grau comercial, Lanxess, RJ, Brasil), divinilbenzeno (DVB) (grau comercial, Nitriflex, RJ, Brasil), material magnético, n-heptano (PA, Vetec, RJ, Brasil - grau de diluição 100\%) e 2-2'- azo-bis-isobutironitrila (AIBN) (grau comercial, recristalizado em metanol, Migquímica, SP, Brasil) 2\% molar em relação ao número de mols totais de monômeros. A fase aquosa (FA) foi composta de poli(álcool vinílico)(PVA) (PA, Kuraray, Japão) $1 \%$ m.v. e de $\mathrm{NaCl}$ (PA, Vetec, RJ, Brasil) 1\% m.v. A fase orgânica foi 
adicionada, sob agitação de 700 rpm, sobre a fase aquosa (razão volumétrica $\mathrm{FO}: \mathrm{FA}=1: 4$ ) e as polimerizações foram conduzidas por $4 \mathrm{~h}$ a $80^{\circ} \mathrm{C}$. O produto final foi lavado exaustivamente com água deionizada aquecida e seco em estufa a $60^{\circ} \mathrm{C}$

\subsection{Caracterização}

Para a confirmação da composição das microesferas magnéticas foi feito FT-IR (Perkin Elmer, modelo Spectrum One). As condições para obtenção dos espectros foram as seguintes: ATR, resolução: $4,0 \mathrm{~cm}^{-1}$, acumulação: 4 . As características magnéticas dos materiais foram analisadas em Magnetômetro de Amostra Vibrante (VSM)(Lake Shore, modelo Série 7400). Foram utilizadas cerca de $0,05 \mathrm{~g}$ de amostra, cujo campo magnético aplicado variou de $-14000 \mathrm{G}$ a $14000 \mathrm{G}$ e o tempo total de cada análise foi de 10 minutos, sendo os dados coletados a cada segundo. A técnica de absorção atômica (AAS) foi utilizada para a determinação do teor de ferro incorporado nos polímeros. Nesta análise, $0,01 \mathrm{~g}$ de amostra foi submetida à digestão por Microondas (Milestone, modelo Start D) com $10 \mathrm{~mL}$ de água régia durante 40 minutos à $180^{\circ} \mathrm{C}$. Após esta etapa, as soluções foram filtradas e, por conseguinte, transferidas para balões volumétricos de $100 \mathrm{~mL}$ onde seu volume foi completado com água deionizada. Em seguida, a absorbância da solução foi medida no equipamento (Varian, modelo AA240) e a concentração de ferro foi determinada com o auxílio de uma curva padrão de absorbância versus concentração de ferro. A estrutura cristalina das amostras foi verificada por difratometria de raios X (Shimadzu, modelo XRD 6000). O aparelho era equipado com tubo de Fe e com um monocromador de grafite. As varreduras foram feitas entre $7-70^{\circ}$ (2-theta) com velocidade do goniômetro de $2^{\circ}$ por minuto. Foi utilizado o programa JADE para a identificação das fases mineralógicas presente nas amostras. A espectroscopia de Mössbauer foi utilizada para identificar o material magnético, sendo eficiente na identificação das fases de $\mathrm{Fe}^{2+}$ ou Fe ${ }^{3+}$, permitindo diferenciar a magnetita e a maghemita. A análise de fases foi medida usando fonte de ${ }^{57} \mathrm{Co} / \mathrm{Rh}$ em transmissão padrão à temperatura ambiente. Os espectros foram coletados em um espectrômetro convencional de 512 canais, na faixa de velocidade de -11 a $11 \mathrm{~mm} / \mathrm{s}$. A calibração da velocidade foi obtida a partir do padrão $\alpha$-Fe. Os ajustes dos espectros foram realizados computacionalmente com sextetos e/ou dubletos simétricos. Os teores de cada fase mineralógica contendo ferro foram calculados com o uso das áreas relativas obtidas nos ajustes espectrais e os valores de ferro total foram obtidos por análise química. $\mathrm{O}$ tamanho e distribuição de tamanho de partículas dos suportes poliméricos magnéticos foram analisados por espalhamento de luz (Malvern, modelo Mastersizer 2000) e a morfologia foi analisada por SEM-EDS (Fei Company, modelo The Inspect 550), onde as microesferas foram aderidas sobre bases metálicas, usando uma fita adesiva dupla-face e aplicou-se uma fina camada de ouro. As amostras metalizadas interagiram com elétrons secundários, em alto vácuo, sob uma tensão de aceleração de $20 \mathrm{kV}$. O equipamento ASAP 2020 (Micromeritics) foi utilizado para a realização das análises de adsorção de nitrogênio pelas amostras com base nas isotermas de adsorção à $-196^{\circ} \mathrm{C}$. Os métodos matemáticos utilizados nesta caracterização foram o Barrett, Joyner, e
Halenda (BJH) para a distribuição do tamanho de poros e o Brunauer, Emmett, e Teller (BET) para a determinação quantitativa da área superficial. Antes de se proceder aos ensaios de adsorção, as amostras foram desgaseificadas a $100^{\circ} \mathrm{C}$ durante 24 horas sob pressão máxima de $925 \mathrm{mmHg}$.

\section{Resultados e Discussão}

O material magnético obtido foi caracterizado por difração de raios X. A fase do óxido de ferro foi identificada nos padrões de difração de raios $\mathrm{X}$ pelos picos nas posições aproximadas de $2 \Theta=22,9^{\circ} ; 38,0^{\circ} ; 45^{\circ} ; 54,9^{\circ}$ e $68,7^{\circ}$ nos planos cristalinos de 472, 270,333, 175 e 90, respectivamente. Entretanto, como os parâmetros de rede de maghemita e magnetita são próximos, os picos de difração também são. Tendo em vista o pequeno tamanho de partícula, que causa um alargamento dos picos, temos então uma sobreposição quase que completa dos picos destas duas fases. Conclui-se que técnica de difração de raios X precisa ser complementada por outra técnica capaz de distinguir os óxidos de ferro constituintes da amostra. Assim, a espectrometria de Mössbauer foi também utilizada para caracterizar os materiais. O espectro Mössbauer (Figura 1) mostra essencialmente dois sextetos que podem ser associados a uma fase ferrimagnetica (área espectral 52\%) e um sexteto largo e mal definido que pode ser associado à goethita (área espectral $48 \%$ ). A fase ferrimagnética é muito provavelmente uma mistura de magnetita e maghemita, sendo a distinção neste tipo de amostra muito difícil de ser realizada. De acordo com a literatura ${ }^{[13]} \mathrm{o}$ armazenamento de magnetita pode levá-la a oxidação topotática em decorrência do tempo. Este processo de oxidação inicia-se na superfície, a qual sugere a existência de um núcleo $\mathrm{Fe}^{2+}$ nas partículas. Assim, o mais provável é que tenha ocorrido a oxidação parcial da magnetita. Foi observado também que o material magnético obtido era fortemente atraído por um ímã.

As microsferas poliméricas magnéticas obtidas foram caracterizadas por FT-IR. As análises de FT-IR realizadas no poli(metacrilato de metila-co-divinilbenzeno) contendo material magnético (P(MMA-co-DVB)-M) mostraram a banda característica das ligações $\mathrm{Fe}-\mathrm{O}$ na faixa de $600-620 \mathrm{~cm}^{-1}$ confirmando a incorporação do material

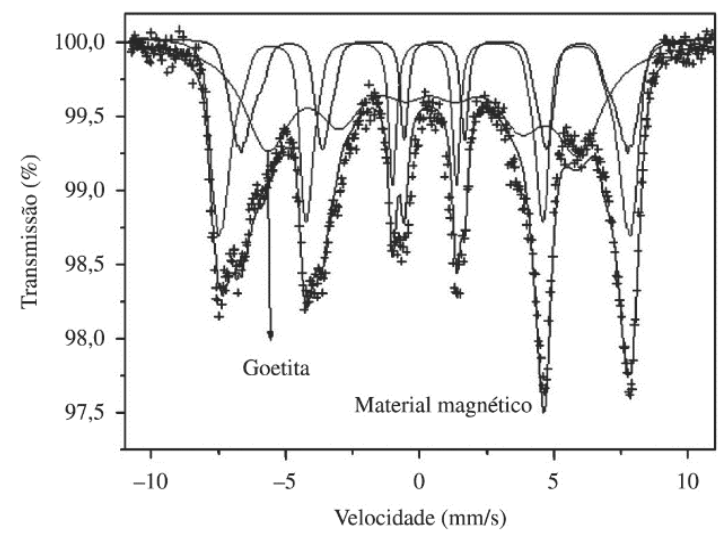

Figura 1. Espectrometria Mössbauer do material magnético sintetizado. 
magnético ao polímero ${ }^{[14]}$. A vibração de alongamento assimétrico na região de $1720 \mathrm{~cm}^{-1}$ indicou a existência da carbonila contida nas microesferas. A vibração simétrica característica do grupo C-O apareceu em $1230 \mathrm{~cm}^{-1}$, os alongamentos do tipo assimétrico e simétrico referente a C-H saturado aparecem nas regiões de 2970 e $2880 \mathrm{~cm}^{-1}$, respectivamente. As vibrações carbono-carbono que se estende dentro do anel (grupos fenila) aparecem na região de $1590 \mathrm{~cm}^{-1}$ e $1410 \mathrm{~cm}^{-1}$. Já a banda em $980 \mathrm{~cm}^{-1}$ foi atribuída aos benzenos dissubstituídos e $750 \mathrm{~cm}^{-1}$ aos benzenos monossubstituídos características do divinilbenzeno.

A Tabela 1 mostra os parâmetros reacionais utilizados na síntese de $\mathrm{P}(\mathrm{MMA}$-co-DVB)-M e os resultados obtidos quanto ao teor de ferro incorporado, área específica, volume dos poros, magnetização de saturação e tamanho de partículas obtidos nos copolímeros. Observa-se diferentes concentrações de óxido de ferro incorporadas no polímero. A amostra sintetizada pela polimerização denominada semi-shaker apresentou uma concentração maior de material magnético incorporado em relação as polimerizações s/semi e semi-balão. Uma explicação plausível, é que na polimerização em um shaker, os monômeros estão mais sujeitos a se volatilizar reduzindo a massa de polímero produzida e, consequentemente, aumentando o teor de óxido no compósito final. A polimerização S/semi e a Semi-balão produziram resultados próximos, quanto ao teor de incorporação de ferro, mostrando que a semissuspensão não influenciou na incorporação do material magnético neste sistema. Já nas variações de material magnético, o aumento da quantidade de material magnético adicionada pode explicar a tendência destes materiais em apresentarem maiores concentrações de óxido de ferro incorporado. Pode-se notar que a variação do teor de DVB não provocou nenhuma alteração significativa na incorporação do material magnético nos copolímeros.

A Figura 2 mostra as micrografias eletrônicas de varredura obtidas dos P(MMA-co-DVB)-M. Na primeira etapa, onde foram utilizadas diferentes tipos de suspensão (Figura 2a-c) observa-se a presença de esferas quebradas ou com deformação em sua esfericidade no tipo de síntese sem semissuspensão (S/ semi)(Figura 2a) e com semissuspensão com shaker (Semishaker) (Figura 2b). A Figura 2c mostra que semissuspensão com auxílio do balão (Semi-balão) foi mais eficiente em manter o formato esférico. É possível que este tipo de síntese tenha homogeneizado melhor a fase orgânica. Yuan et al. ${ }^{[15]}$ também citam que a partir do uso da semissuspensão há a obtenção de uma mistura reacional altamente viscosa antecipadamente. É provável que, durante a Semi-shaker (Figura $2 b$ ), a pouca dispersão associada ao aumento da viscosidade do meio tenha limitado a reação. A deficiência na homogeneização da fase orgânica também poderia auxiliar na formação de clusters de material magnético durante a síntese. As Figuras 2d-f, apresentam a micrografia eletrônica dos copolímeros sintetizados com 5\% (Fe5\%) (Figura 2d), 10\% (Fe10\%) (Figura 2e) e 15\% de material magnético adicionado (Fe15\%) (Figura 2f). A síntese que utilizou 5\% de material magnético (Figura 2d) apresentou partículas com boa morfologia esférica por toda extensão do material. Nas sínteses que utilizaram 10 e $15 \%$ de material magnético, nota-se uma maior quantidade de partículas com perda de esfericidade. A Figura $2 \mathrm{~g}$ mostra $\mathrm{o}$ copolímero sintetizado com $3 \%$ de DVB. Este material apresentou pouquíssimas esferas e, em geral, o material permaneceu sem forma definida. É possível que a pouca quantidade de DVB, nesta síntese, não tenha favorecido a suspensão por completo. Como o DVB utilizado é de grau comercial (com aproximadamente $55 \%$ DVB), o teor de DVB expresso acima (3\%) não reflete a quantidade efetiva de agente reticulante, podendo não levar a formação de ligações cruzadas, e ficar na forma de grupos vinilas pendentes não-polimerizáveis ${ }^{[16]}$. Outra explicação poderia ser o aumento da polaridade do meio com a diminuição do teor de DVB. O aumento da polaridade reduz a tensão interfacial e, consequentemente, as gotas de monômero com o pré-polímero tendem a se deformar mais. As Figuras $2 \mathrm{~h}$ e 2i (DVB10\% e DVB20\%, respectivamente) apresentam copolímeros magnéticos com formato esférico bem definido.

A Figura 3 mostra as micrografias de microscopia eletrônica de varredura das partes externa e interna das microesferas contendo $10 \%$ de DVB e $20 \%$ de DVB, juntamente com os mapas de composição de ferro. Podem ser observados, nas Figuras 3a, 3c, 3e, 3g, pequenos aglomerados de partículas na superfície das microesferas poliméricas. Estes aglomerados são de material magnético, como pode

Tabela 1. Influência de alguns parâmetros reacionais na síntese de microesferas poliméricas magnéticas ( P(MMA-co-DVB)-M).

\begin{tabular}{|c|c|c|c|c|c|c|c|c|c|}
\hline Amostra & $\begin{array}{c}\text { Tipo de } \\
\text { polimerização } \\
\text { em suspensão }\end{array}$ & $\begin{array}{c}\text { Teor de } \\
\text { DVB ( } \% \\
\text { molar) }\end{array}$ & $\begin{array}{c}\text { Material } \\
\text { magnético } \\
\text { adicionado } \\
(\% \text { m.m)1 }\end{array}$ & $\begin{array}{c}\text { Material } \\
\text { magnético } \\
\text { Incorporado } \\
(\% \text { m.m }) 2\end{array}$ & $\begin{array}{c}\text { Área } \\
\text { específica } \\
\left(\mathbf{m}^{2} \cdot \mathrm{g}^{-1}\right)\end{array}$ & $\begin{array}{c}\text { Volume } \\
\text { dos poros } \\
\left(\mathrm{cm}^{3} / \mathrm{g}\right)\end{array}$ & $\begin{array}{c}\text { Magnetização } \\
\text { de Saturação } \\
\left(\mathrm{emu} \cdot \mathrm{g}^{-1}\right)\end{array}$ & $\begin{array}{c}\text { Magnetização } \\
\text { remanescente } \\
\left(\mathrm{emu} . \mathrm{g}^{-1}\right)\end{array}$ & $\begin{array}{c}\text { Faixa de } \\
\text { tamanho } \\
\text { de } \\
\text { partículas } \\
(\mu \mathrm{m}) \\
\end{array}$ \\
\hline 1 & S/ semi3 & & & $5,82 \pm 0,03$ & n.d.6 & n.d. & 5,28 & 0,26 & $8-1200$ \\
\hline 2 & Semi-shaker4 & 10 & 5,0 & $14,63 \pm 0,02$ & n.d. & n.d. & 7,22 & 0,32 & $5-120$ \\
\hline 3 & Semi-balão5 & & & $4,13 \pm 0,02$ & 48,30 & 0,353 & 4,76 & 0,21 & $20-104$ \\
\hline 4 & & & 5,0 & $4,13 \pm 0,02$ & 48,30 & 0,353 & 4,76 & 0,21 & $20-104$ \\
\hline 5 & Semi-balão & 10 & 10 & $8,54 \pm 0,01$ & n.d. & n.d. & 8,73 & 0,38 & $10-320$ \\
\hline 6 & & & 15 & $13,5 \pm 0,08$ & n.d. & n.d. & 16,19 & 0,68 & $5-104$ \\
\hline 7 & & 3 & & $4,28 \pm 0,02$ & n.d. & n.d. & 4,55 & 0,28 & $8-120$ \\
\hline 8 & Semi-balão & 10 & 5 & $4,13 \pm 0,02$ & 48,30 & 0,353 & 4,76 & 0,21 & $20-104$ \\
\hline 9 & & 20 & & $4,36 \pm 0,03$ & 40,43 & 0,233 & 6,56 & 0,34 & $10-120$ \\
\hline
\end{tabular}

${ }^{1}$ Material magnético adicionado em função da massa total da fase orgânica; ${ }^{2}$ Material magnético incorporado no copolímero obtido por absorção atômica; ${ }^{3} 0,3$ mols totais $\left(0,27\right.$ mols MMA, 0,03 mols de DVB), $1 \%$ m.v PVA, $1 \%$ m.v NaCl; ${ }^{4} 80 \mathrm{u}, 30$ minutos. $50{ }^{\circ} \mathrm{C} ;{ }^{5} 700 \mathrm{rpm}, 30$ minutos, $50{ }^{\circ} \mathrm{C} ;{ }^{6}$ n.d. $=$ não determinado. 

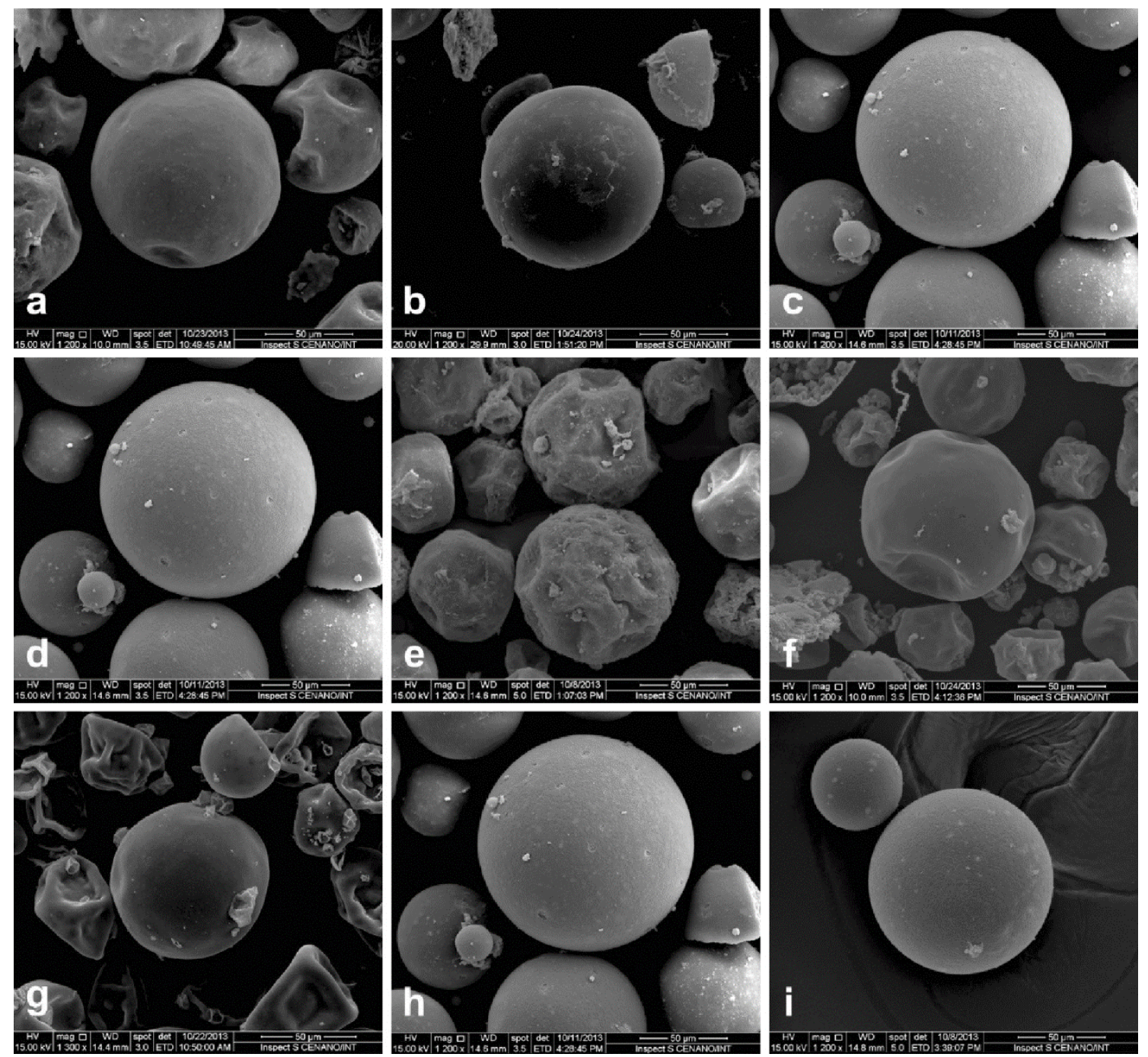

Figura 2. Micrografias eletrônicas de varredura dos $\mathrm{P}(\mathrm{MMA}-\mathrm{co}-\mathrm{DVB})-\mathrm{M}$ : E a) sem pré, b) pré-shaker, c) pré-balão; d) $\mathrm{Fe} 5 \%$, e) Fe $10 \%$, f) Fe15\% g) DVB 3\%, h) DVB 10\% e i) DVB 20\% (Aumento: $1.200 \mathrm{X}$ ).

ser comprovado pelos respectivos mapas de composição de ferro (Figuras 3b, d, f, h). Nota-se também, através destas figuras, que a incorporação das partículas magnéticas pelos copolímeros ocorreu de maneira heterogênea, tanto na superfície, quanto no interior das microesferas.

Estes resultados corroboram com os resultados de teor de ferro incorporado nos copolímeros apresentados na Tabela 1, que demonstraram haver uma irregularidade na incorporação do material magnético no polímero. As microesferas fraturadas, apresentadas nas Figuras 3c e 3g, mostram que suas partes internas são mais porosas que suas superfícies externas. O uso de um não solvente (n-heptano) provocou um aumento dos tamanhos dos macroporos no interior das microesferas. Já em suas superfícies externas, mostradas nas Figuras 3a e 3d, os domínios poliméricos se apresentam como estruturas compactas e sem forma definida. Estas diferenças morfológicas entre a superfície externa e o interior das microesferas são características comuns de polímeros reticulados macroporosos sintetizados através de polimerização em suspensão. A maior compactação dos domínios poliméricos na superfície do que no interior das microesferas tem sido atribuída a um efeito de compressão devido à tensão interfacial entre a fase orgânica e a fase aquosa durante a polimerização em suspensão ${ }^{[17]}$. Foram obtidas as isotermas de adsorção de nitrogênio pela análise de ASAP dos P(MMA-co-DVB)-M sintetizados com 10\% DVB e $20 \%$ DVB (Figura $4 a$ ). A forma das isotermas de ambos os copolímeros apresentaram-se como do tipo IV, referente a sólidos com poros razoavelmente grandes (mesoporos), onde a histerese está relacionada com diferenças entre os processos de adsorção e dessorção. Sabe-se que o tipo de isoterma é função do efeito do tamanho do poro sobre o fenômeno de adsorçãa ${ }^{[18]}$. O estudo do fenômeno de adsorção é feito com o objetivo de se obter informações sobre a área específica e a estrutura porosa do sólido. Assim, as análises mostraram que com o aumento da concentração de DVB de 10 a $20 \%$, houve uma diminuição na área específica (BET) $\left(\right.$ de $48,3 \mathrm{~m}^{2} / \mathrm{g}$ para $\left.40,43 \mathrm{~m}^{2} / \mathrm{g}\right)$, no tamanho dos 

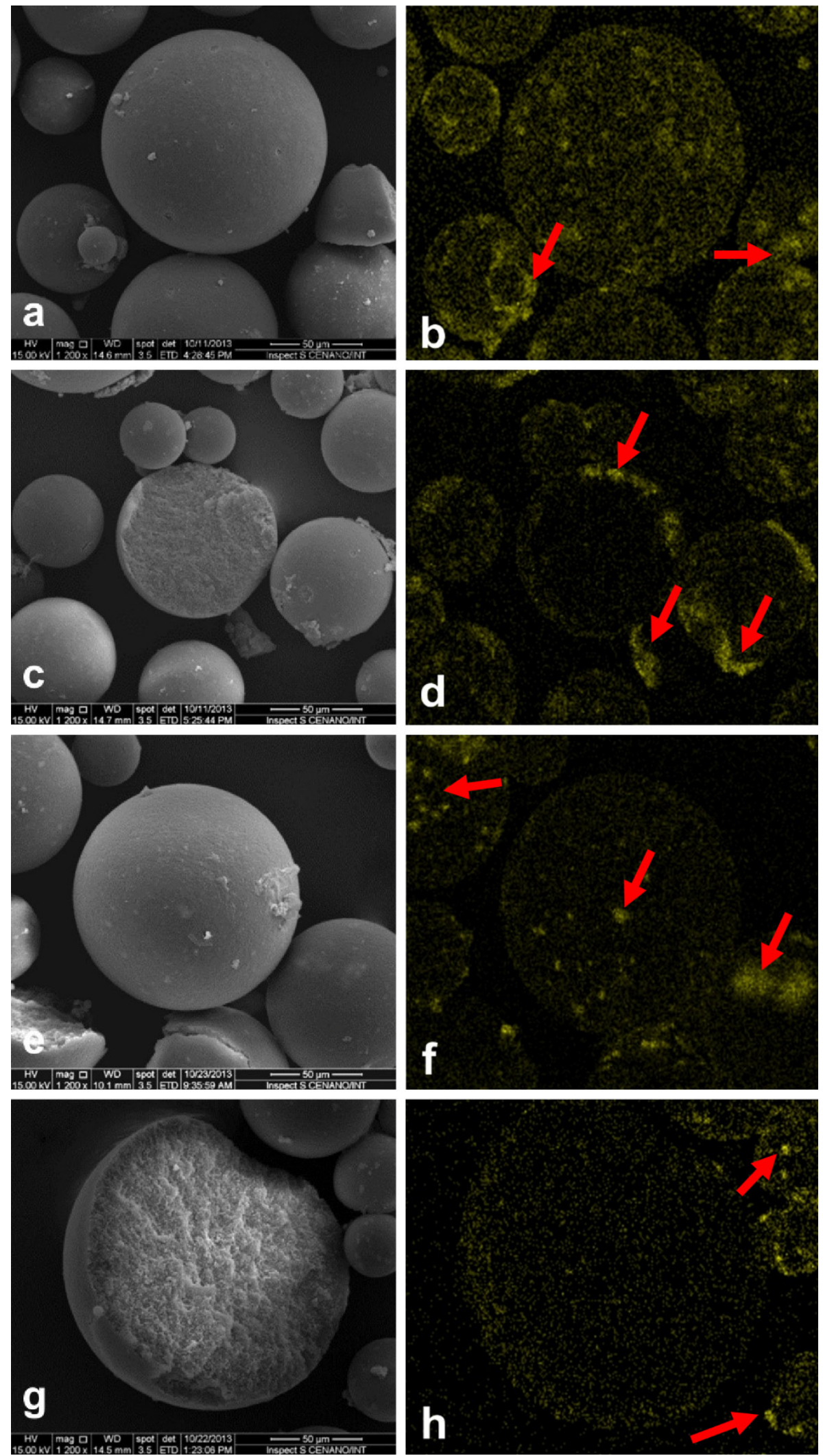

Figura 3. Micrografias eletrônicas de varredura de P(MMA-co-DVB)-M : DVB 10\% - a) Parte externa , b) Mapa de composição de ferro da parte externa, c) Parte interna , d) Mapa de composição de ferro da parte interna e DVB 20\% - e) Parte externa, f) Mapa de composição de ferro parte externa, g) Parte interna, h) Mapa de composição de ferro da parte interna (Aumento: 1.200X); ( $<$ ) Aglomerados de óxido de ferro. 
(a)

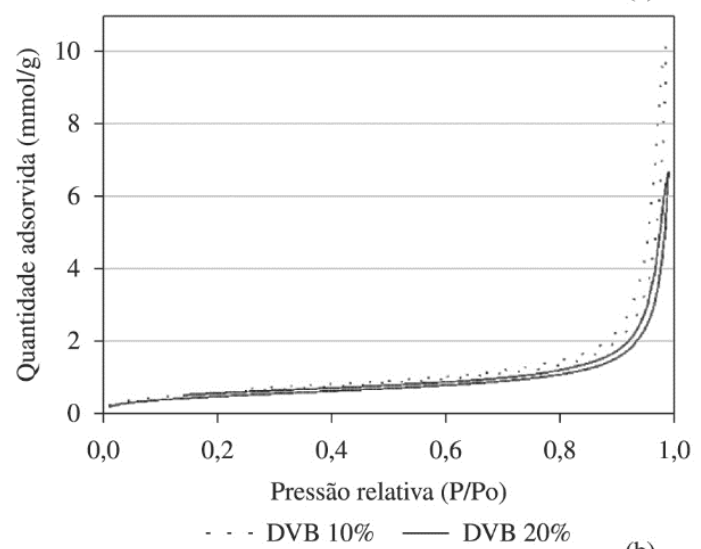

(b)

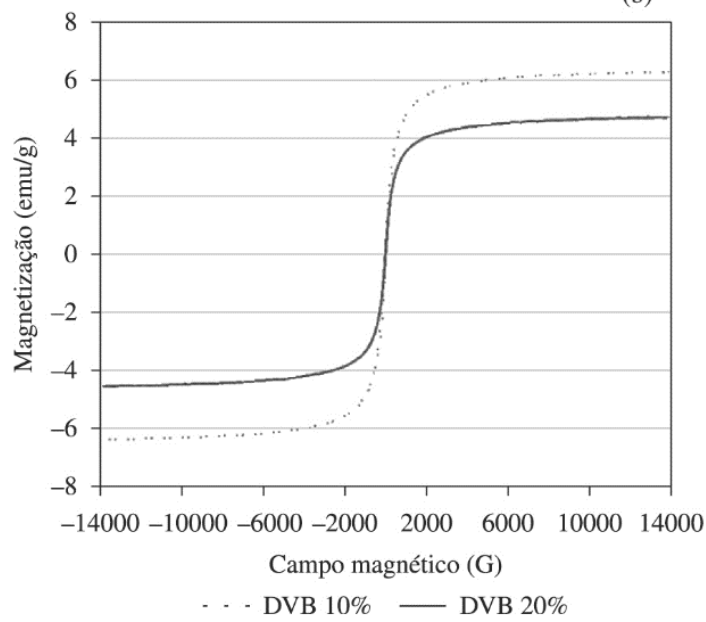

Figura 4. Caracterizações de P(MMA-co-DVB)-M obtidos com 10 e $20 \%$ de DVB a) Isotermas de adsorção e dessorção de nitrogênio; b) Curvas de magnetização de saturação obtidas por VSM.

poros (BJH) (de $270 \AA$ para $220 \AA$ ) e no volume dos poros $\left(0,353 \mathrm{~cm}^{3} / \mathrm{g}\right.$ para $\left.0,233 \mathrm{~cm}^{3} / \mathrm{g}\right)$ destes copolímeros. A redução da área específica com o aumento do teor de DVB pode ser explicada por uma redução do grau de separação de fases durante a polimerização. Considerando que o aumento de DVB implica em uma diminuição do teor de MMA e o uso de heptano, como diluente, a interação do heptano com cadeias poliméricas menos polares é melhor do que com cadeias poliméricas mais polares. A literatura também relata que existem alguns sistemas poliméricos à base de monômeros polares reticulados com DVB que apresentam uma relação entre a porosidade e o teor de agente de reticulação diferente de sistemas mais comuns como STY-DVB. Este comportamento deve-se as diferenças nas reatividades e nas propriedades termodinâmicas do monômero polar em relação ao reticulador DVB. Assim, o polímero formado nas fases iniciais de polimerização pode conter mais unidades de DVB do que na mistura de monômeros. Consequentemente, aumentando-se o conteúdo do agente de reticulação é possível que ocorra uma diminuição significativa da porosidade do produto final ${ }^{[19]}$. A distribuição de tamanho de partículas dos copolímeros foi determinada pela técnica de espalhamento de luz a fim de se determinar o tamanho médio das partículas, bem como, a sua distribuição de tamanhos. Seus resultados também são apresentados na Tabela 1. De um modo geral, o uso de AIBN como iniciador e de PVA como estabilizante proporcionou uma distribuição de tamanho de partículas estreita e a obtenção de uma maior quantidade de partículas na faixa de $50-90 \mu \mathrm{m}$. Estes resultados corroboram com outros resultados da literatura ${ }^{[5,9]}$. Na etapa onde diferentes tipos de suspensão foram utilizados, foi possível verificar que a utilização da técnica de semissuspensão com balão mostrou-se mais eficiente na manutenção da uniformidade do tamanho das microesferas neste sistema, e que o uso da semissuspensão levou a produção de partículas poliméricas com distribuição de tamanho mais estreita do que uma polimerização em suspensão convencional. Estes resultados estão, também, de acordo com a literatura ${ }^{[4,10]}$. No estudo onde se utilizou diferentes concentrações de DVB foi verificado que o aumento do teor de DVB (20\%) também deslocou de maneira significativa o tamanho de partículas para faixas de tamanho maiores em relação ao polímero com $10 \%$ de DVB. O aumento do tamanho de partícula com o aumento do teor de DVB também pode ser explicado pela redução da polaridade e a aumento da tensão interfacial durante a polimerização em suspensão. Os resultados de magnetização de saturação $\left(M_{s}\right)$ e magnetização remanescente $\left(M_{r}\right)$ dos copolímeros de P(MMA-co-DVB)-M sintetizados são apresentadas na Tabela 1. De um modo geral todos os copolímeros apresentaram uma boa resposta ao campo magnético e $\mathrm{M}_{\mathrm{r}}$ próximas a zero, indicando um comportamento superparamagnético. Os copolímeros apresentaram $\mathrm{M}_{\mathrm{s}}$ na faixa de 4,7-16,0 emu/g. Os resultados de $\mathrm{M}_{\mathrm{s}}$ e teor de ferro incorporado (Tabela 1) reforçam o comportamento heterogêneo da incorporação do ferro nas partículas poliméricas descrito anteriormente. As curvas de magnetização de saturação dos copolímeros magnéticos sintetizados com 10 e $20 \%$ de DVB também são apresentadas na Figura 4b. Estes materiais não apresentaram ciclos de histerese, fenômeno que causa o atraso entre a densidade de fluxo magnético (B) e o campo magnético $(\mathrm{H})$, indicando também o comportamento superparamagnético. A literatura apresenta valores de magnetização de saturação para microesferas poliméricas magnéticas na faixa de $1,9-17,0 \mathrm{emu} / \mathrm{g}^{[1,23,36]}$. Lee et al. ${ }^{[20]}$ obtiveram magnetizações de saturação na faixa de 1,2 e $4,0 \mathrm{emu} / \mathrm{g}$ que, segundo os autores esses resultados seriam adequados para a utilização destes materiais como resinas de troca iônica com propriedades magnéticas. Yuan et al. ${ }^{[21]}$ também obtiveram materiais com magnetizações de saturação próximas de $8,0 \mathrm{emu} / \mathrm{g}$, que segundo os autores, já seria um valor excelente para utilizar as microesferas em processos catalíticos.

\section{Conclusões}

Foram obtidas, com sucesso, microesferas poliméricas magnéticas à base de MMA e DVB (P(MMA-co-DVB)-M), contendo material magnético tanto na superfície quanto no interior da microesfera. As partículas obtidas apresentaram tamanhos médios menores e distribuição de tamanho de partículas mais estreita que suspensões convencionais e comportamento superparamagnético com magnetizações de saturação entre 4,7-16,0 emu/g. O uso em conjunto de AIBN, PVA e semissuspensão em balão proporcionou a obtenção de 
esferas com tamanhos menores do que em sistemas à base de MMA conhecidos. Os resultados deste estudo que melhor proporcionaram a produção de microesferas com tamanhos menores, distribuição de tamanhos mais homogêneos e com boas propriedades magnéticas foram as condições reacionais com a utilização da semissuspensão com balão, adição de 5\% de material magnético e 10 e $20 \%$ de DVB. As microesferas magnéticas sintetizadas neste trabalho têm potencial para serem modificadas e aplicadas como resinas de troca iônica ou suportes catalíticos.

\section{Agradecimentos}

Os autores agradecem à FAPERJ, ao CNPq e a CAPES (Edital 04/CII-2008, REDE NANOBIOTEC - BRASIL) pelo apoio financeiro. A autora Jacira A. Castanharo agradece a bolsa de doutorado recebida da REDE NANOBIOTEC - BRASIL (CAPES). Os autores agradecem também ao Prof. José Carlos Pinto (COPPE/ UFRJ) pelas análises de Espalhamento de luz e as empresas Nitriflex e Migquímica, pelas doações de DVB e AIBN, respectivamente.

\section{Referências}

1. Ma, Z., Guan, Y., Liu, X., \& Liu, H. (2005). Preparation and characterization of micron-sized non-porous magnetic polymer microspheres with immobilized metal affinity ligands by modified suspension polymerization. Journal of Applied Polymer Science, 96(6), 2174-2180. http://dx.doi.org/10.1002/ app.21688.

2. Yang, C., Liu, H., Guan, Y., Xing, J., Liu, J., \& Shan, G. (2005). Preparation of magnetic poly(methylmethacrylatedivinylbenzene-glycidylmethacrylate) microspheres by spraying suspension polymerization and their use for protein adsorption. Journal of Magnetism and Magnetic Materials, 293(1), 187192. http://dx.doi.org/10.1016/j.jmmm.2005.01.060.

3. Costa, C. N., Costa, M. A. S., Santa-Maria, L. C., Souza, F. G. Jr, $\&$ Michel, R. (2012). Síntese e caracterização de copolímeros à base de metacrilato de metila e divinilbenzeno com propriedades magnéticas. Polímeros: Ciência e Tecnologia, 22(3), 260-266. http://dx.doi.org/10.1590/S0104-14282012005000042.

4. Machado, F., Lima, E. L., \& Pinto, J. C. (2007). Uma revisão sobre os processos de polimerização em suspensão. Polímeros: Ciência e Tecnologia, 17(2), 166-179. http://dx.doi.org/10.1590/ S0104-14282007000200016.

5. Castanharo, J. A., Mello, I. L., Santa-Maria, L. C., Costa, M. A., Silva, M. A., \& Oliveira, M. G. (2012). Preparação e caracterização de microesferas poliméricas magnéticas à base de estireno, divinilbenzeno e acetato de vinila. Polímeros: Ciência e Tecnologia, 22(3), 303-309. http://dx.doi.org/10.1590/ S0104-14282012005000038.

6. Souza, F. S., Costa, M. A. S., Santa-Maria, L. C., Mello, I. L., Silva, M. R., \& Hui, W. S. (2013). Síntese e caracterização de copolímeros reticulados à base de estireno, divinilbenzeno $\mathrm{e}$ metacrilato de metila com propriedades magnéticas. Polímeros: Ciência e Tecnologia, 23(1), 82-90. http://dx.doi.org/10.1590/ S0104-14282013005000004.

7. Bombard, A. J. F. (2005). Suspensões magneto-reológicas de pós de ferro carbonilo - um estudo da influência das propriedades magnéticas e do tamanho de partículas (Tese de doutorado). Universidade Estadual de Campinas, Campinas.
8. Salazar-Alvarez, G. (2004). Synthesis, characterization and applications of iron oxide nanoparticles (Tese de doutorado). Royal Institute of Technology, Suécia.

9. Santa-Maria, L. C., Leite, M. C. A. M., Costa, M. A. S., Ribeiro, J. M. S., Senna, L. F., \& Silva, M. R. (2004). Characterization of magnetic microspheres based on network styrene and divinylbenzene copolymers. Materials Letters, 58(24), 30013006. http://dx.doi.org/10.1016/j.matlet.2004.05.028.

10. Conceição, B. M., Costa, M. A. S., Santa-Maria, L. C., Silva, M. R., \& Wang, S. H. (2011). A study of the initiator concentration's effect on styrene-divinylbenzene polymerization with iron particles. Polímeros: Ciência e Tecnologia, 21(5), 409-415. http://dx.doi.org/10.1590/S0104-14282011005000070.

11. Koneracká, M., Kopčanský, P., Antalík, M., Timko, M., Ramchand, C. N., Lobo, D., Mehta, R. V., \& Upadhyay, R. V. (1999). Immobilization of proteins and enzymes to fine magnetic particles. Journal of Magnetism and Magnetic Materials, 201(1-3), 427-430. http://dx.doi.org/10.1016/ S0304-8853(99)00005-0.

12. Guo, Z., Bai, S., \& Sun, Y. (2003). Preparation and characterization of immobilized lipase on magnetic hydrophobic microspheres. Enzyme and Microbial Technology, 32(7), 776-782. http:// dx.doi.org/10.1016/S0141-0229(03)00051-6.

13. Mincheva, R., Stoilova, O., Penchev, H., Ruskov, T., Spirov, I., Manolova, N., \& Rashkov, I. (2008). Synthesis of polymer-stabilized magnetic nanoparticles and fabrication of nanocomposite fibers thereof using electrospinning. European Polymer Journal, 44(3), 615-627. http://dx.doi.org/10.1016/j. eurpolymj.2007.11.001.

14. Karaagac, O., Kockar, H., Beyaz, S., \& Tanrisever, T. (2010). A simple way to synthesize superparamagnetic iron oxide nanoparticles in air atmosphere: iron ion concentration effect. IEEE Transactions on Magnetics, 46(12), 3978-3983. http:// dx.doi.org/10.1109/TMAG.2010.2076824.

15. Yuan, H. G., Kalfas, G., \& Ray, W. R. (1991). Suspension polymerization. Journal of Macromolecular Science, Part C: Polymer Reviews, 31(2-3), 215-299. http://dx.doi. org/10.1080/15321799108021924.

16. Bartholin, M., Boissier, G., \& Dubois, J. (1981). Styrenedivinylbenzene copolymers, 3. Revisited IR analysis. Die Makromolekulare Chemie, 182(7), 2075-2085. http://dx.doi. org/10.1002/macp.1981.021820719.

17. Rabelo, D. (1993). Formação da estrutura porosa em copolímeros à base de estireno e divinilbenzeno (Tese de doutorado). Universidade Federal do Rio de Janeiro, Rio de Janeiro.

18. Teixeira, V. G., Coutinho, F. M., \& Gomes, A. S. (2001). Principais métodos de caracterização da porosidade de resinas à base de divinilbenzeno. Quimica Nova, 24(6), 808-818. http://dx.doi.org/10.1590/S0100-40422001000600019.

19. Okay, O. (2000). Macroporous copolymer networks. Progress in Polymer Science, 25(6), 711-779. http://dx.doi.org/10.1016/ S0079-6700(00)00015-0.

20. Lee, Y., Rho, J., \& Jung, B. (2003). Preparation of magnetic ion-exchange resins by the suspension polymerization of styrene with magnetite. Journal of Applied Polymer Science, 89(8), 2058-2067. http://dx.doi.org/10.1002/app.12365.

21. Yuan, D., Zhang, Q., \& Dou, J. (2010). Supported nanosized palladium on superparamagnetic composite microspheres as an efficient catalyst for Heck reaction. Catalysis Communications, 11(7), 606-610. http://dx.doi.org/10.1016/j.catcom.2010.01.005.

Enviado: Fev. 11, 2014

Revisado: Jul. 18, 2014 Aceito: Nov. 13, 2014 